\title{
Structure and Properties of Dual-doped PEDOT:PSS Multilayer Films
}

\author{
Dalius Jucius ${ }^{* 1} \mathbb{D}$, Algirdas Lazauskas ${ }^{1}$, Viktoras Grigaliūnas ${ }^{1}$, Rimantas Gudaitis ${ }^{1}$, Asta Guobiene $\dot{e}^{1}$, \\ Igoris Prosyčevas ${ }^{1}$, Brigita Abakevičiene $\dot{e}^{1}$, Mindaugas Andrulevičius ${ }^{1}$ (D) \\ ${ }^{1}$ Institute of Materials Science, Kaunas University of Technology, Kaunas, Lithuania
}

Received: February 14, 2019; Revised: May 09, 2019; Accepted: October 16, 2019

\begin{abstract}
Poly(3,4-ethylenedioxythiophene):poly(styrenesulfonate) (PEDOT:PSS) is the most successful transparent conductive polymer widely used in functional organic devices. However, its potential in fabrication of highly conductive electrodes still has not been fully explored. This paper is an attempt to fill the existing gap of knowledge on possible post-treatments of dimethyl sulfoxide (DMSO)-doped PEDOT:PSS thin films. In the present study, surface morphology, optical and electrical properties of untreated and sulfuric acid treated DMSO-doped PEDOT:PSS multilayers on the glass coverslip substrates are examined. It is demonstrated that short-time sulfuric acid treatment enhances electrical and optical properties, and smoothens surface of the transparent conductive films. Enhancement of electrical conductivity by more than $30 \%$ can be associated with increase in doping level, removal of excess PSS and accompanied shape alterations of the conjugated PEDOT chains. Acid treatment also results in smoother transmittance spectra and a slight improvement of optical transparency. Hence, sulfuric acid post-treatment can be considered as a simple and inexpensive technique for the complementary doping of DMSO-doped PEDOT:PSS films with the aim to develop the more effective transparent electrodes.
\end{abstract}

Keywords: PEDOT:PSS, DMSO, sulfuric acid post-treatment, electrical conductivity, optical properties.

\section{Introduction}

Transparent conductive thin films nowadays are widely used in various optoelectronic devices such as displays, touch screens, light emitting diodes, photovoltaic cells, etc. Main requirements for such films are low electrical resistance and high optical transmittance in visible region. In the past few years, the commonly used benchmark material for transparent conductive films is indium tin oxide (ITO) which is distinguished for a very high electric conductivity reaching up to $10^{4} \mathrm{~S} \mathrm{~cm}^{-1}$ and excellent optical transmittance exceeding $80 \%{ }^{1}$. However, the use of ITO is restricted by complex processing, toxicity, limited lifetime of the electrodes and expensiveness of raw indium ${ }^{2,3}$. Therefore, alternative materials such as other conducting oxides, graphene, carbon nanotubes, nanowires, nanocomposites and conducting polymers are being investigated as possible ITO replacements for the production of less expensive devices with longer lifetimes ${ }^{1,4}$.

Increasing demand for flexible transparent electrodes makes compatible with large-area mass production conductive polymers (CP) an especially promising class of materials ${ }^{5,6}$. So far, various CPs have been investigated including polypyrrole (PPy), polyaniline (PANI), polythiophene (PT), poly(3,4ethylenedioxythiophene) (PEDOT), trans-polyacetylene, and poly(p-phenylene vinylene) (PPV). Among them, solution-processable poly(3,4-ethylenedioxythiophene)/ poly(4-styrenesulfonate) (PEDOT:PSS) is the most popular. Colloidal dispersion of the PEDOT:PSS in water can be easily spin-coated, solution-casted or printed on various substrates. Despite their high quality, films prepared from PEDOT:PSS aqueous solutions cannot be directly used as transparent electrodes since their electrical conductivity is below $1 \mathrm{~S} \mathrm{~cm}^{-1}$ 7-9.

Conductivity of PEDOT:PSS can be significantly improved by optimizing polymer inter-chain coupling and removal of excess amount of insulating PSS component ${ }^{10}$. For this purpose, addition of organic compound, such as ethylene glycol (EG), dimethyl sulfoxide (DMSO), dimethylformamide (DMF), ionic liquid or anionic surfactant is very beneficial and can enhance the conductivity by two or three orders of magnitude ${ }^{11-14}$. Enhancement in conductivity of the films, associated with morphological and compositional changes, such as grain growth, polymer chain expansion, phase separation and PSS removal, can also be achieved by post-deposition treatments with heat, salts, cosolvents and acids ${ }^{15-17}$. Recently reported results demonstrated that treatment with concentrated sulfuric acid can enhance the conductivity of PEDOT:PSS films to more than $4 \times 10^{3} \mathrm{~S} \mathrm{~cm}^{-1}{ }^{17}$.

Although numerous studies on enhancement of PEDOT:PSS electrical conductivity have been published, there is still a lack of knowledge about possible post- 
treatments of doped PEDOT:PSS transparent conducting electrodes. To fill this gap, we report on sulfuric acid treatment of DMSO-doped PEDOT:PSS multilayers with the aim to improve properties of the films. It is demonstrated that short-time sulfuric acid treatment further enhances electrical and optical properties of DMSO-doped PEDOT:PSS transparent conductors and smoothens surface of the films.

\section{Experimental}

The water dispersions of PEDOT:PSS colloids were synthesized by oxidative polymerization of EDOT (SigmaAldrich) in the presence of the PSS $\left(M_{w}=75,000 \mathrm{~g} / \mathrm{mol}\right.$, Sigma-Aldrich). The EDOT (7.31 g) and PSS (5.85 g) were mixed in deionized water $(1000 \mathrm{ml})$ containing $9.8 \mathrm{~g}$ of $\mathrm{Na}_{2} \mathrm{~S}_{2} \mathrm{O}_{8}$ as an oxidant and $2.0 \mathrm{~g}$ of $\mathrm{FeSO}_{4} 7 \mathrm{H}_{2} \mathrm{O}$ as a catalytic agent. The oxidative polymerization was carried out at $20^{\circ} \mathrm{C}$ for $24 \mathrm{~h}$ under vigorous stirring by a mechanical stirrer in $\mathrm{a}_{2}$ stream at a rate of $3 \mathrm{~L} / \mathrm{min}$ to prevent oxidation from the dissolved oxygen. Afterwards, the iron, sulfate and sodium ions were removed by cation exchange and anion exchange resins.

Thin films were produced by spin-coating water dispersions of PEDOT:PSS colloids containing 5\% of DMSO on a glass substrates at $2000 \mathrm{rpm}$ for $30 \mathrm{~s}$ and dried on a hot plate at 80 ${ }^{\circ} \mathrm{C}$ for $2 \mathrm{~min}$. Post-treatment of the films was performed by immersing PEDOT:PSS coated glass substrates into concentrated $\mathrm{H}_{2} \mathrm{SO}_{4}$ for several seconds followed by rinsing with deionized water and drying under compressed $\mathrm{N}_{2}$. Multilayers were formed by coating up to three layers of PEDOT:PSS and acid post-treatment after the deposition of each layer.

Surface morphology ofDMSO-doped PEDOT:PSS multilayers was investigated using atomic force microscopy (AFM). AFM experiments were carried out in air at room temperature using a NanoWizard III atomic force microscope (JPK Instruments AG), while data was analyzed using SurfaceXplorer and JPK SPM Data Processing software. The AFM images were collected using a V-shaped silicon cantilever (spring constant of $3 \mathrm{~N} / \mathrm{m}$, tip curvature radius of $10.0 \mathrm{~nm}$ and the cone angle of $20^{\circ}$ ) operating in a contact mode. Thickness of DMSO-doped PEDOT:PSS multilayers before and after sulfuric acid treatment was determined using AFM profile images.

Wettability of the films was characterized by measuring static contact angle of deionized water at standard room conditions. Small drop of water (about $5 \mu \mathrm{l}$ ) was dropped onto the surface of the film from the height of $8 \mathrm{~mm}$ using microsyringe. Image of the drop was let to stabilize for 10 seconds after the dropping before recording with PCconnected digital camera. Contact angle was measured at five different positions of each sample. The left and right contact angles of each drop were estimated by a public domain Java image processing program ImageJ $1.40 \mathrm{~g}$. Estimation of the contact angles was made using method based on B-spline snakes (active contours) freely available as DropSnake plugin for ImageJ.
Raman spectra were recorded using inVia Raman spectrometer (Renishaw) equipped with CCD camera and confocal microscope $(50 \times$ objective). The Raman spectra were excited with $532 \mathrm{~nm}$ radiation of semiconductor green laser at $5 \%$ output power in order to avoid damage of the sample. The 2400 lines $/ \mathrm{mm}$ grating was used to record the Raman spectra.

Optical properties of the films were evaluated by measuring UV-vis transmittance spectra. Measurements were carried out using a fiber optic UV/VIS/NIR Avantes AvaSpec-2048 spectrometer (spectral range (SR): 172-1100 nm, resolution $1.4 \mathrm{~nm}$ ) and a light source AvaSpec-DHc (SR: 200-2500 nm). Electrical properties were characterized by sheet resistance measured using the four point probe technique.

\section{Results and Discussion}

Spin coating of PEDOT:PSS+DMSO layers on the glass coverslip substrates resulted in the formation of relatively uniform, defect-free films. The average thickness per layer before sulfuric acid treatment was $50 \pm 2 \mathrm{~nm}$. Short sulfuric acid treatment led to about $4 \mathrm{~nm}$ thinning per layer of the film.

Evolution of surface morphology with the number of untreated and sulfuric acid treated PEDOT:PSS+DMSO layers was examined by AFM. Figure 1 shows characteristic AFM topographical images acquired in air using AC mode. Summary of the roughness parameters is presented in Table 1. It is evident that evenly distributed granular surface structures with the lateral dimensions in the range of 300$500 \mathrm{~nm}$ and height of some tens of $\mathrm{nm}$ are specific for all six samples regardless of the acid treatment and number of the layers. The surfaces of the films are dominated by the peaks with skeweness $\left(R_{s k}\right)$ value from 1.65 to 2.54 and have a leptokurtoic distribution of morphological features (relatively many high peaks and low valleys) with kurtosis $\left(R_{k u}\right)$ values from 5.17 to 10.95 . With increase in number of the layers, surfaces of the films become less asymmetric and less spiky. Roughness of the films increases with the number of layers. A mean height of the surface structures $\left(Z_{\text {mean }}\right)$ and root mean square roughness $\left(R_{q}\right)$ for untreated single-layer film (Figure 1a) was determined to be 5.85 $\mathrm{nm}$ and $5.10 \mathrm{~nm}$, respectively. Whereas for untreated trilayer film (Figure 1c), $Z_{\text {mean }}$ increased to $14.22 \mathrm{~nm}$ and $R_{q}$ increased to $10.19 \mathrm{~nm}$. Such increase in roughness of spin coated multilayers can be associated with cumulative effect of granularity originated from underlying glass substrate. A similar tendency was also observed for sulfuric acid treated multilayers (Figures $1 a_{1}-1 c_{1}$ ). On the other hand, surface of the acid treated films was considerably smoother, with $Z_{\text {mean }}$ of $4.54 \mathrm{~nm}$ and $R_{q}$ of $2.01 \mathrm{~nm}$ for acid treated singlelayer film, and $Z_{\text {mean }}$ of $5.96 \mathrm{~nm}$ and $R_{q}$ of $4.37 \mathrm{~nm}$ for acid treated tri-layer film. This finding is in a good agreement with previous report on sulfuric acid vapor treatment of PEDOT:PSS, attributing decrease in film roughness to the reorientation of PEDOT and PSS chains ${ }^{18}$. Surface 
roughness reduction of transparent organic electrodes by a simple acid treatment prior to spin-coating of another layer can be considered as a very positive result because organic semiconductor devices are in general very sensitive to the surface roughness and $R_{q}$ is a critical factor to be accounted for to achieve the best device performance. In our case, sulfuric acid treatment has also resulted in a slight decrease in lateral dimensions of granular surface structures and, at the same time, small-scale bumps with the diameter of some tens of nanometers were highlighted on the surface of each granule (Figure 1 $\mathrm{a}_{1}$ ). Highlighted nanoroughness can be attributed to the removal of excess PSS from the surface of film and emergence of PEDOTrich nanobumps ${ }^{19}$.
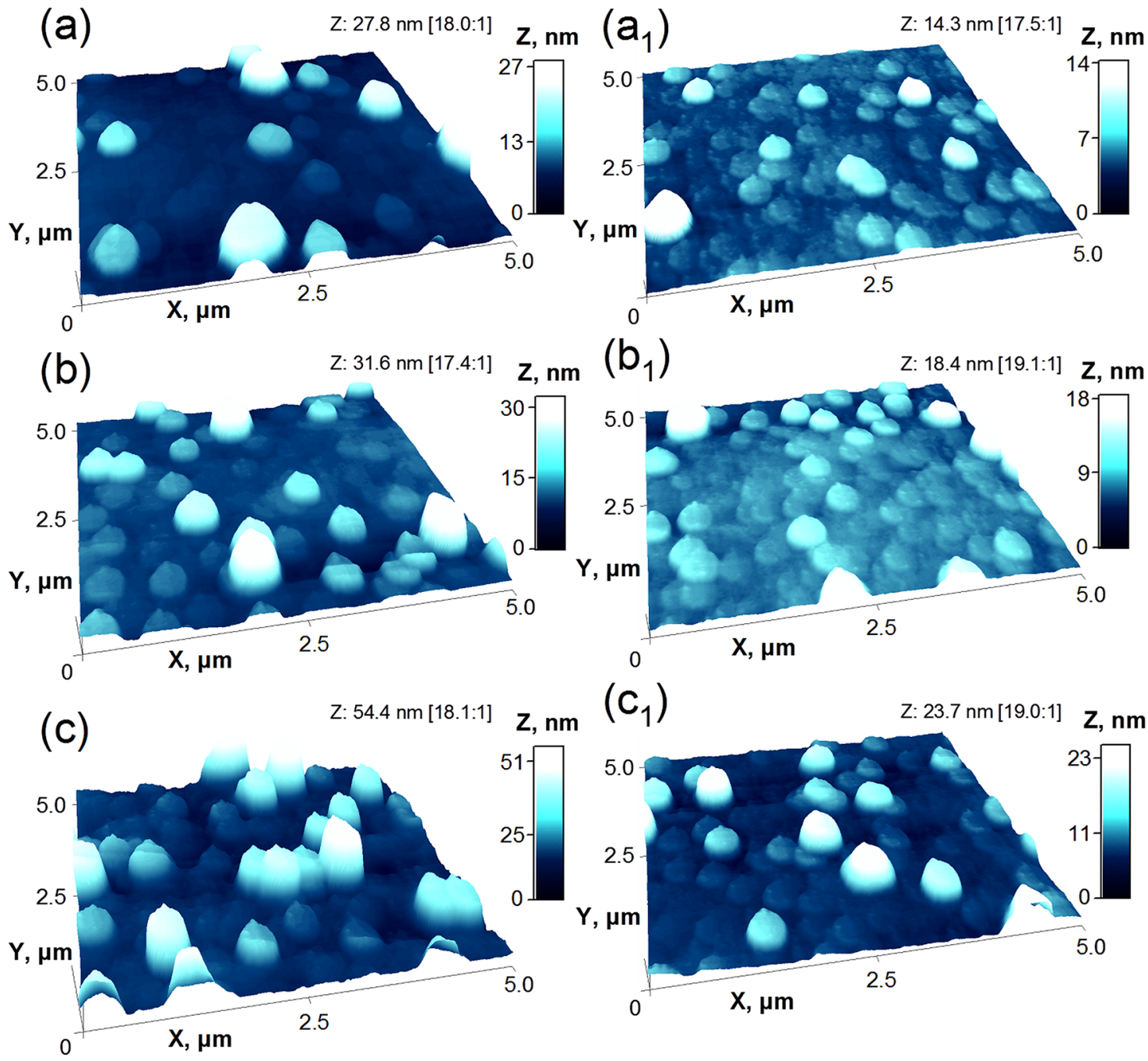

$\left(c_{1}\right)$

Z: $23.7 \mathrm{~nm}[19.0: 1]$

$\mathbf{Z}, \mathbf{n m}$

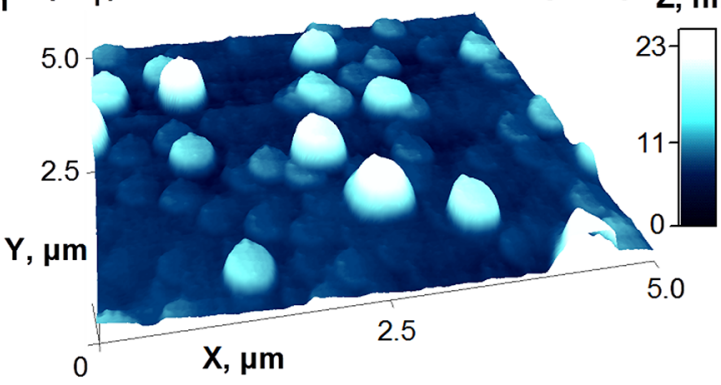

Figure 1. Characteristic AFM topographical images of (a, b, c) untreated and (a1, b1, c1) sulfuric acid treated PEDOT:PSS+DMSO (a, a1) single-layer, (b, b1) bi-layer, and (c, c1) tri-layer films

Table 1. Summary of the roughness parameters for untreated and sulfuric acid treated PEDOT:PSS+DMSO films

\begin{tabular}{lcccc}
\hline \multirow{2}{*}{ Sample } & \multicolumn{3}{c}{ Roughness parameters } \\
\cline { 2 - 5 } & $\mathbf{R q}, \mathbf{n m}$ & $\mathbf{R s k}$ & $\mathbf{R k u}$ & $\mathbf{Z m e a n , ~} \mathbf{n m}$ \\
\hline Single-layer PEDOT:PSS + DMSO & 5.10 & 2.28 & 7.67 & 5.85 \\
Bi-layer PEDOT:PSS + DMSO & 5.39 & 2.21 & 8.07 & 10.08 \\
Tri-layer PEDOT:PSS + DMSO & 10.19 & 1.66 & 5.17 & 14.22 \\
Single-layer PEDOT:PSS + DMSO / H2SO4 & 2.01 & 2.54 & 10.95 & 4.54 \\
Bi-layer PEDOT:PSS + DMSO / H2SO4 & 2.74 & 1.88 & 7.84 & 7.24 \\
Tri-layer PEDOT:PSS + DMSO / H2SO4 & 4.37 & 1.97 & 6.73 & 5.96 \\
\hline
\end{tabular}


Detailed investigation of structural arrangement between PEDOT and PSS in the films was carried out by recording and examining AFM phase images, which are known to be very sensitive to variations in chemical and mechanical properties of the sample and can help to distinguish different compounds. Representative AFM phase images of untreated and sulfuric acid treated PEDOT:PSS+DMSO single-layer films are presented in Figure 2. The bright regions of the phase images correspond to the stiffer PEDOT-rich grains inducing a higher phase shift, whereas dark regions correspond to the softer PSS-rich matrix ${ }^{20}$. In the case of untreated PEDOT:PSS film, PEDOT-rich grains with lateral dimensions on the order of tens $\mathrm{nm}$ are already significantly aggregated due to the influence of DMSO. However, these grains are almost completely embedded into the PSS matrix. Sulfuric acid treatment results in noticeably increased phase contrast which can be interpreted in terms of more pronounced phase separation and partial removal of PSS from the surface of the film.

Wettability changes of PEDOT:PSS+DMSO films were evaluated by measuring static water contact angle. Dependence of the contact angle on the number of layers before and after sulfuric acid treatment is presented in Figure 3. Surface of untreated PEDOT:PSS+DMSO films was hydrophilic with average water contact angle increasing from $17^{\circ}$ for single-layer film to $20^{\circ}$ for tri-layer film. Small decrease in wetting with increase in the number of layers can be explained by the changes in surface roughness of the films. Sulfuric acid treatment resulted in significant increase of the contact angle. However, in contrast to the untreated films, it was no clear dependence of contact angle on the number of layers. Regardless the number of the layers, contact angle on sulfuric acid treated surfaces was close to $40^{\circ}$. Substantial increase in contact angle can be associated with decrease in surface energy of sulfuric acid treated films as a result of the partial removal of hydrophilic PSS from the surface of acid treated PEDOT:PSS+DMSO films.

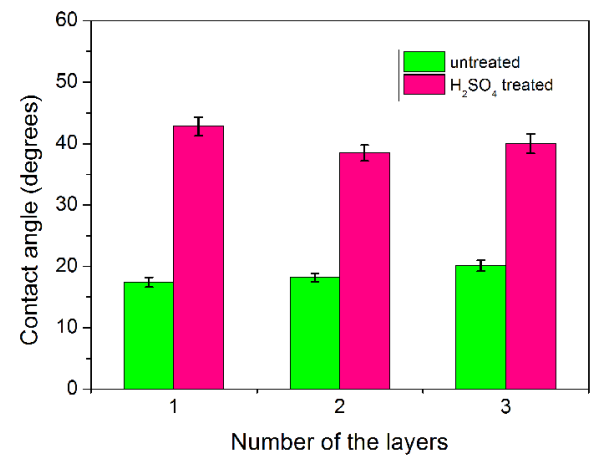

Figure 3. Water contact angle on untreated and sulfuric acid treated DMSO-doped PEDOT:PSS films. Each column of the histogram is plotted with error bar representing the standard deviation

Figure 4a shows Raman spectrum of untreated PEDOT:PSS+DMSO tri-layer film under green light excitation with a wavelength of $532 \mathrm{~nm}$. The most intense band at $1428 \mathrm{~cm}^{-1}$ is attributed to PEDOT and corresponds to the $\mathrm{C}_{\alpha}=\mathrm{C}_{\beta}$ symmetric stretching vibration of five-membered thiophene ring originating from neutral parts existing between the localized elementary excitations ${ }^{21,22}$. Absence of the shoulder at about $1442 \mathrm{~cm}^{-1}$, corresponding to the symmetric stretching modes of benzoid $\mathrm{C}_{\alpha}=\mathrm{C}_{\beta}$ components, indicates domination of quinoid structures due to the conformational transformation of the PEDOT chains from coiled to linear or expanded-coiled. The bands located at 1486 and $1569 \mathrm{~cm}^{-1}$ are associated with thiophene rings in the middle and at the end of the PEDOT chains. These bands are assigned to the asymmetric stretching vibration of $\mathrm{C}_{\alpha}=\mathrm{C}_{\beta}$; the band at 1537 $\mathrm{cm}^{-1}$ is associated with the splitting of these vibrations ${ }^{23}$. The $\mathrm{C}_{\beta}-\mathrm{C}_{\beta}$ stretching deformations and $\mathrm{C}_{\alpha}-\mathrm{C}_{\alpha}$ inter-ring stretching vibrations of $\mathrm{PEDOT}$ are assigned to the bands centered at (a)

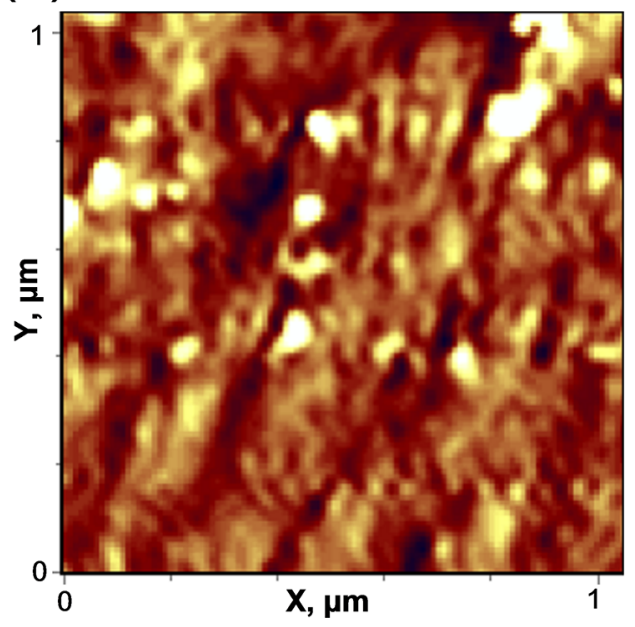

(b)

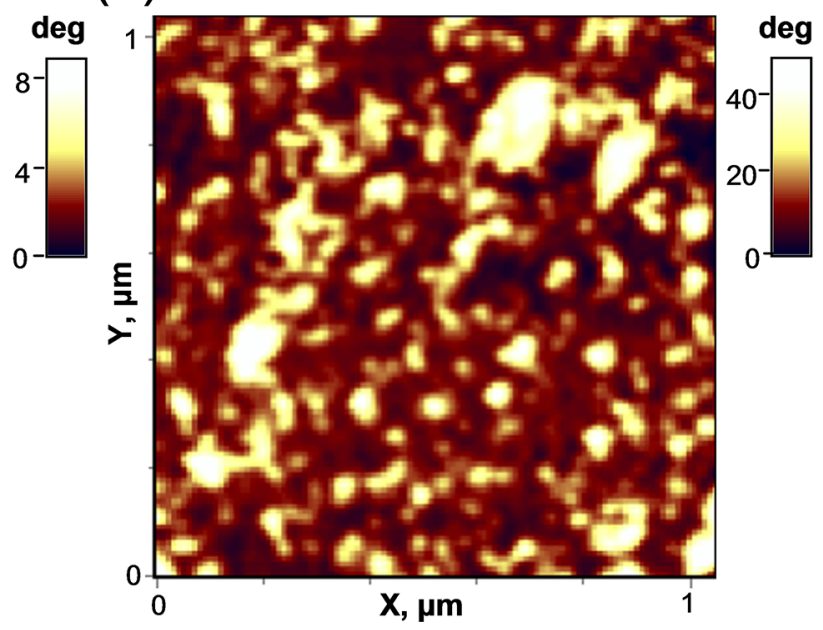

Figure 2. AFM phase images of (a) untreated and (b) sulfuric acid treated PEDOT:PSS+DMSO single-layer films 
1367 and $1256 \mathrm{~cm}^{-1} 24$. The bands at 1124 and $1098 \mathrm{~cm}^{-1}$ correspond to the vibrational modes of PSS component ${ }^{25}$, and the bands at 988,855 and $577 \mathrm{~cm}^{-1}$ - to the oxyethylene ring deformation-vibrations ${ }^{26,27}$. The symmetric $\mathrm{C}-\mathrm{S}-\mathrm{C}$ deformation is assigned to the band centered at $702 \mathrm{~cm}^{-1}$. The band at $439 \mathrm{~cm}^{-1}$ is assigned to the doping of PEDOT by the $\mathrm{SO}_{3}^{-}$ion from PSS units ${ }^{28}$.

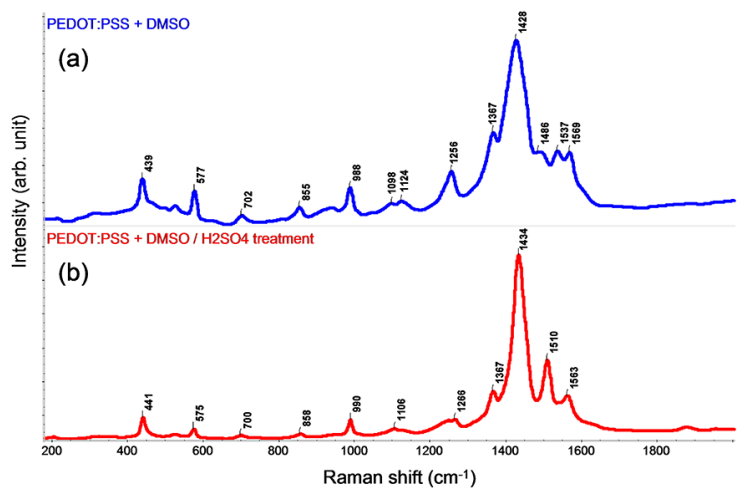

Figue 4. Raman spectra of (a) untreated PEDOT:PSS+DMSO and (b) sulfuric acid treated PEDOT:PSS+DMSO tri-layer films

After sulfuric acid treatment of PEDOT:PSS+DMSO tri-layer film, the band at $1428 \mathrm{~cm}^{-1}$ becomes narrower and blue shifted to $1434 \mathrm{~cm}^{-1}$ (Figure $4 \mathrm{~b}$ ). Peak shifting to higher wavenumbers can be associated with increase in the film doping level due to the incorporation of sulfate anions during acid post-treatment. This effect is related to the degree of backbone deformation during oxidation to polarons and bi-polarons and the associated transitions between quinoid and benzoid structures. Decrease in bandwidth can serve as an extra evidence of further transformation of PEDOT structure to quinoid ${ }^{29-31}$. Disappearance of the bands at 1486 and $1537 \mathrm{~cm}^{-1}$ and appearance of new intensive band at 1510 $\mathrm{cm}^{-1}$ can be caused by rearrangement of PEDOT chains as a result of the oxidizing action of present in $\mathrm{HSO}_{4}^{-}$ions oxygen over not balanced by PSS thiophene rings. Moreover, disappearance of the band at $1124 \mathrm{~cm}^{-1}$ indicates that a large portion of the remaining PSS-rich regions in DMSO-doped PEDOT:PSS film is removed during the acid treatment. Decrease of intensity for the peak at $990 \mathrm{~cm}^{-1}$ also can be related to decrease in the PSS concentration. The band at $439 \mathrm{~cm}^{-1}$ is shifted to $441 \mathrm{~cm}^{-1}$ after the doping of sulfate and bisulfate anions originating in PEDOT from sulfuric acid.

Optical properties of DMSO-doped PEDOT:PSS films before and after sulfuric acid treatment were investigated by measuring UV-visible transmittance spectra (Figure 5). Spectrum of the bare glass coverslip substrate showed 93 $\%$ average transparency of the substrates in the $350-800 \mathrm{~nm}$ wavelength range. Spin-coating of thin PEDOT:PSS layers slightly decreased the transmittance. Optical transparency at violet and blue light region $(350-500 \mathrm{~nm})$ decreased from 87 to $77 \%$ for single-layer and tri-layer PEDOT:PSS films, respectively. Decrease in transparency at red light region (620$750 \mathrm{~nm}$ ) was more significant and varied from 84 to $67 \%$ for single-layer and tri-layer PEDOT:PSS films, respectively. Decrease in the transmittance when moving from the violet to the red region of the spectrum is reported to be typical for PEDOT:PSS films ${ }^{32}$. In our case, this phenomenon was more pronounced for thicker tri-layer films where the blue color of the film became quite visible. However, single-layer films demonstrated very good transparency in entire visible region with only $3 \%$ decrease in transmittance at the red end of the spectrum. Short sulfuric acid treatment even resulted in a slight improvement of optical transparency. 1-2 \% improvement of the transmittance was observed for single-layer and bi-layer PEDOT:PSS films in the $350-500 \mathrm{~nm}$ wavelength range, whereas for tri-layer films the maximum increase in transmittance reaching 3-4 \% was registered within wavelengths of 500 to $750 \mathrm{~nm}$. Moreover, the acid treatment generally resulted in smoother transmittance spectra of PEDOT:PSS films. Obtained positive changes in transmittance might be associated firstly with significant smoothing of the surface and slight thinning of the acid treated films, and secondly with PSS removal and structural rearrangement of PEDOT:PSS chains.

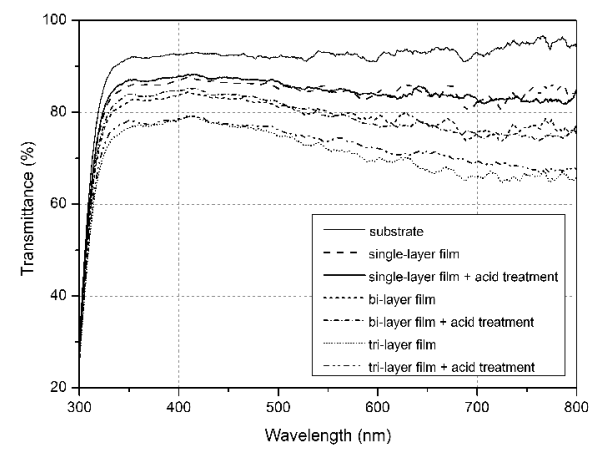

Figure 5. Optical transmittance of untreated and sulfuric acid treated DMSO-doped PEDOT:PSS films

Dependence of average sheet resistance on the number of pristine and sulfuric acid treated DMSO-doped PEDOT:PSS layers is presented in Figure 6. The average sheet resistance for the pristine single-layer films, due to the conformational changes caused by the strong dipole-dipole or dipole-charge interaction between DMSO and PEDOT:PSS chains, and DMSO-induced shielding of electrostatic interactions between PEDOT and PSS, was as low as $710 \Omega / \mathrm{sq}$ and it was further decreased by adding more layers. Sulfuric acid treatment was very effective for enhancement of electrical conductivity. The average sheet resistance after the acid treatment decreased from 710 to $450 \Omega / \mathrm{sq}$ for single-layer films and from 210 to $140 \Omega / \mathrm{sq}$ for tri-layer films.

Conducting mechanism of the DMSO-doped sulfuric acid treated PEDOT:PSS films is evidently associated with 


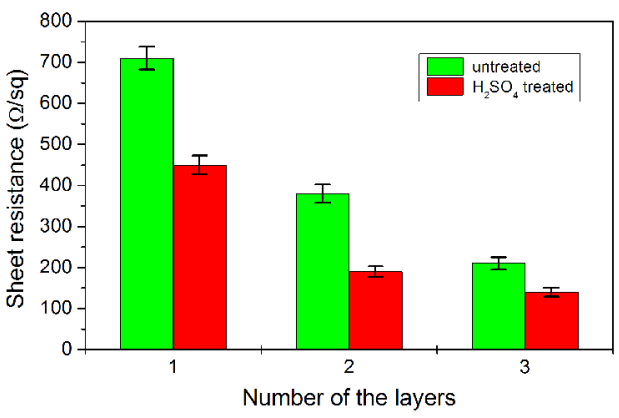

Figure 6. Sheet resistance of the pristine and sulfuric acid treated DMSO-doped PEDOT:PSS films. Each column of the histogram is plotted with error bar representing the standard deviation

an increase of doping level. The doping level of the pristine films depends on the amount of charge carriers created by the single-charged $\mathrm{SO}_{3}^{-}$ions from PSS segments. Acid treatment results in the substitution of some PSS- counter ions by bisulfate and sulfate ions. Bisulfate ions do not change doping level as they are also single-charged, whereas the sulfate ions are double-charged and can stimulate generation of bipolaron carriers instead of polarons in the PEDOT chains ${ }^{33}$. Moreover, additional doping of PEDOT chains can be an outcome of the oxidizing action of present in bisulfite ions oxygen over thiophene rings that are not balanced by PSS ${ }^{-34}$. Hydrogen cations originated from the dissociation of sulfuric acid can further interact with PSS to form polystyrene sulfonic acid, which is subsequently removed by rinsing the films in deionized water ${ }^{28}$. This removal in our case is confirmed by Raman analysis and contact angle measurements. So, increase in doping level, removal of excess PSS and accompanied shape alterations of the conjugated PEDOT chains can be considered as the main factors enhancing electrical conductivity of sulfuric acid treated DMSO-doped PEDOT:PSS films.

\section{Conclusions}

In the present study, surface morphology, optical and electrical properties of untreated and sulfuric acid treated DMSO-doped PEDOT:PSS multilayers on the glass coverslip substrates are examined. It is demonstrated that short-time sulfuric acid treatment enhances electrical and optical properties, and smoothens surface of the transparent conductive films. Underlying mechanism for the enhancement of electrical conductivity by more than $30 \%$ can be associated with increase in doping level due to the substitution of some PSS $^{-}$counter ions by sulfate ions, and additional doping of PEDOT chains due to the oxidizing action of present in bisulfite ions oxygen over not balanced by PSS- thiophene rings. Moreover, changes in surface morphology and electrical conductivity are evidently caused by the removal of excess
PSS and accompanied shape alterations of the conjugated PEDOT chains. Removal of hydrophilic PSS from the surface of acid treated films is apparently confirmed by substantial increase in water contact angle and characteristic changes in Raman spectra and AFM images. The single-layer films, with the thickness of about $50 \mathrm{~nm}$, demonstrated very good transparency in entire visible region with only $3 \%$ decrease in transmittance at the red end of the spectrum. Short sulfuric acid treatment even resulted in smoother transmittance spectra and a slight improvement of optical transparency. It can be concluded that sulfuric acid post-treatment can be used as a simple and inexpensive mean for the complementary doping of DMSO-doped PEDOT:PSS transparent conductive electrodes with the aim to further improve their electrical conductivity to be better suited for optoelectronic devices.

\section{Acknowledgment}

This research was funded by the European Social Fund under the No. 09.3.3-LMT-K-712 "Development of Competences of Scientists, other Researchers and Students through Practical Research Activities" measure. Grant No. 09.3.3-LMT-K-712-01-0074.

\section{References}

1. Hofmann AI, Cloutet E, Hadziioannou G. Materials for Transparent Electrodes: From Metal Oxides to Organic Alternatives. Advanced Electronic Materials. 2018;4(10):1700412.

2. Cao W, Li J, Chen H, Xu J. Transparent electrodes for organic optoelectronic devices: a review. Journal of Photonics for Energy. 2014;4(1):040990.

3. Kulkarni GU, Kiruthika S, Gupta R, Rao KDM. Towards low cost materials and methods for transparent electrodes. Current Opinion in Chemical Engineering. 2015;8:60-68.

4. O'Connor B, Haughn C, An KH, Pipe KP, Shtein M. Transparent and conductive electrodes based on unpatterned, thin metal films. Applied Physics Letters. 2008;93(22):223304.

5. Zhu H, Shen Y, Li Y, Tang J. Recent advances in flexible and wearable organic optoelectronic devices. Journal of Semiconductors. 2018;39(1):011011.

6. Fabretto MV, Evans DR, Mueller M, Zuber K, Hojati-Talemi $\mathrm{P}$, Short RD, et al. Polymeric Material with Metal-Like Conductivity for Next Generation Organic Electronic Devices. Chemistry of Materials. 2012;24(20):3998-4003.

7. Le TH, Kim Y, Yoon H. Electrical and Electrochemical Properties of Conducting Polymers. Polymers. 2017;9(4):150.

8. Yan H, Jo T, Okuzaki H. Potential application of highly conductive and transparent poly(3,4-ethylenedioxythiophene)/ poly(4-styrenesulfonate) thin films to touch screen as a replacement for indium tin oxide electrode. Polymer Journal. 2011;43:662-665.

9. Li Y. Organic Optoelectronic Materials (Lecture Notes in Chemistry 91). Cham: Springer International Publishing; 2015 . 
10. Tan L, Zhou H, Ji T, Huang L, Chen Y. High conductive PEDOT via post-treatment by halobenzoic for high-efficiency ITOfree and transporting layer-free organic solar cells. Organic Electronics. 2016;33:316-323.

11. Sarker AK, Kim J, Wee BH, Song HJ, Lee Y, Hong JD, et al. Hydroiodic acid treated PEDOT:PSS thin film as transparent electrode: an approach towards ITO free organic photovoltaics. RSC Advances. 2015;5(64):52019-52025.

12. Lee I, Kim GW, Yang M, Kim TS. Simultaneously Enhancing the Cohesion and Electrical Conductivity of PEDOT:PSS Conductive Polymer Films using DMSO Additives. ACS Applied Materials \& Interfaces. 2016;8(1):302-310.

13. Wei Q, Mukaida M, Naitoh Y, Ishida T. Morphological Change and Mobility Enhancement in PEDOT:PSS by Adding Cosolvents. Advanced Materials. 2013;25(20):2831-2836.

14. Döbbelin M, Marcilla R, Salsamendi M, Pozo-Gonzalo C, Carrasco PM, Pomposo JA, et al. Influence of Ionic Liquids on the Electrical Conductivity and Morphology of PEDOT:PSS Films. Chemistry of Materials. 2007;19(9):2147-2149.

15. Huang J, Miller PF, de Mello JC, de Mello AJ, Bradley DDC. Influence of thermal treatment on the conductivity and morphology of PEDOT/PSS films. Synthetic Metals. 2003;139(3):569-572.

16. Nitta A, Imamura Y, Kawahara K, Takeda K. Examination of Treatment Methods for a PEDOT:PSS Transparent Conductive Film Produced Using an Inkjet Method. Advances in Materials Physics and Chemistry. 2017;7(8):311-322.

17. Wen Y, Xu J. Scientific Importance of Water-Processable PEDOT-PSS and Preparation, Challenge and New Application in Sensors of Its Film Electrode: a review. Journal of Polymer Science Part A: Polymer Chemistry. 2017;55(7):1121-1150.

18. Kim J, Jang JG, Hong JI, Kim SH, Kwak J. Sulfuric acid vapor treatment for enhancing the thermoelectric properties of PEDOT:PSS thin-films. Journal of Materials Science: Materials in Electronics. 2016;27(6):6122-6127.

19. Zabihi F, Xie Y, Gao S, Eslamian M. Morphology, conductivity, and wetting characteristics of PEDOT:PSS thin films deposited by spin and spray coating. Applied Surface Science. 2015;338:163-177.

20. Lang U, Müller E, Naujoks N, Dual J. Microscopical Investigations of PEDOT:PSS Thin Films. Advanced Functional Materials. 2009;19(8):1215-1220.

21. Xu B, Gopalan SA, Gopalan AI, Muthuchamy N, Lee KP, Lee JS, et al. Functional solid additive modified PEDOT: PSS as an anode buffer layer for enhanced photovoltaic performance and stability in polymer solar cells. Scientific Reports. 2017;7:45079.

22. Ji T, Tan L, Hu X, Dai Y, Chen Y. A comprehensive study of sulfonated carbon materials as conductive composites for polymer solar cells. Physical Chemistry Chemical Physics. 2015;17(6):4137-4145.
23. Singh V, Arora S, Arora M, Sharma V, Tandon RP. Characterization of doped PEDOT: PSS and its influence on the performance and degradation of organic solar cells. Semiconductor Science and Technology. 2014;29(4):045020.

24. Nguyen TP, de Vos SA. An investigation into the effect of chemical and thermal treatments on the structural changes of poly(3,4-ethylenedioxythiophene)/polystyrenesulfonate and consequences on its use on indium tin oxide substrates. Applied Surface Science. 2004;221(1-4):330-339.

25. Chang SH, Chiang CH, Kao FS, Tien CL, Wu CG. Unraveling the Enhanced Electrical Conductivity of PEDOT:PSS Thin Films for ITO-Free Organic Photovoltaics. IEEE Photonics Journal. 2014;6(4):8400307.

26. Garreau S, Louarn G, Buisson JP, Froyer G, Lefrant S. In Situ Spectroelectrochemical Raman Studies of Poly $(3,4-$ ethylenedioxythiophene)(PEDT). Macromolecules. 1999;32(20):6807-6812.

27. Park H, Lee SH, Kim FS, Choi HH, Cheong IW, Kim JH. Enhanced thermoelectric properties of PEDOT: PSS nanofilms by a chemical dedoping process. Journal of Materials Chemistry A. 2014;2(18):6532-6539.

28. Sarat Kumar SR, Kurra N, Alshareef HN. Enhanced high temperature thermoelectric response of sulphuric acid treated conducting polymer thin films. Journal of Materials Chemistry C. 2016;4(1):215-221.

29. Chiu WW, Travaš-Sejdić J, Cooney RP, Bowmaker GA. Spectroscopic and conductivity studies of doping in chemically synthesized poly(3,4-ethylenedioxythiophene). Synthetic Metals. 2005;155(1):80-88.

30. Lee SH, Park H, Kim S, Son W, Cheong IW, Kim JH. Transparent and flexible organic semiconductor nanofilms with enhanced thermoelectric efficiency. Journal of Materials Chemistry A. 2014;2(20):7288-7294.

31. Pehlivaner Kara MO, Frey MW. Effects of solvents on the morphology and conductivity of poly(3,4-ethylenedioxythio phene):Poly(styrenesulfonate) nanofibers. Journal of Applied Polymer Science. 2014;131(11):40305.

32. Hau SK, Yip HL, Zou J, Jen AKY. Indium tin oxide-free semitransparent inverted polymer solar cells using conducting polymer as both bottom and top electrodes. Organic Electronics. 2009;10(7):1401-1407.

33. Reyes-Reyes M, Cruz-Cruz I, López-Sandoval R. Enhancement of the Electrical Conductivity in PEDOT: PSS Films by the Addition of Dimethyl Sulfate. The Journal of Physical Chemistry C. 2010;114(47):20220-20224.

34. Cruz-Cruz I, Reyes-Reyes M, López-Sandoval R. Formation of polystyrene sulfonic acid surface structures on poly $(3,4$ ethylenedioxythiophene): Poly(styrenesulfonate) thin films and the enhancement of its conductivity by using sulfuric acid. Thin Solid Films. 2013;531:385-390. 